\title{
Probing the gluon TMDs with quarkonia
}

\author{
Jean-Philippe Lansberg* \\ IPN Orsay, Paris Sud U., CNRS/IN2P3, Université Paris-Saclay, F-91406 Orsay, France \\ E-mail: Jean-Philippe.Lansberg@in2p3.fr
}

\section{Cristian Pisano}

Dipartimento di Fisica, Università di Cagliari, and INFN, Sezione di Cagliari Cittadella

Universitaria, I-09042 Monserrato (CA), Italy

\section{Florent Scarpa}

IPN Orsay, Paris Sud U., CNRS/IN2P3, Université Paris-Saclay, F-91406 Orsay, France \& Van Swinderen Institute for Particle Physics and Gravity, University of Groningen, Nijenborgh 4, 9747 AG Groningen, The Netherlands

\section{Marc Schlegel}

Department of Physics, New Mexico State University, Las Cruces, NM 88003, USA

We briefly review how quarkonium hadroproduction can be used to access the polarised and unpolarised gluon TMDs.

XXVI International Workshop on Deep-Inelastic Scattering and Related Subjects (DIS2018)

16-20 April 2018

Kobe, Japan

${ }^{*}$ Speaker. 


\section{Introduction}

Transverse-Momentum-Dependent (TMD) factorisation [1, 2, 3, 4] allows one to study the impact of the polarisation of partons with nonzero transverse momentum -even inside unpolarised hadrons - via the appearance of azimuthal modulations. At hadron colliders, these modulations mostly come from gluons and these new phenomena are encapsulated in the distribution $h_{1}^{\perp g}\left(x, \boldsymbol{k}_{T}^{2}\right)$ of linearly-polarised gluons [5]. In practice, one expects $\cos 2 \phi(\cos 4 \phi)$ modulations in the yield of two-particle final states. These follow from single (double) gluon-helicity flips in gluon-fusion processes. The correlation between the polarisation of these gluons and their transverse momentum can also alter the transverse-momentum spectrum of the produced system. One example is that of the Brout-Englert-Higgs $H^{0}$ boson $[6,7]$ produced after double gluon-helicity flips.

We review here how some processes involving quarkonium production can help experimentally determine these poorly known gluon distributions. First, we start with the production of a pseudoscalar quarkonium which is very similar to the $H^{0}$ case. Second, we discuss the production of a $J / \psi$ or $\Upsilon$ in association with a photon or a $Z^{0}$ boson. Third, we report on the unique case of di- $J / \psi$ production which is expected to exhibit the largest possible $\cos 4 \phi$ modulations and for which data exist allowing for the first extraction of the unpolarised gluon distributon $f_{1}^{g}\left(x, \boldsymbol{k}_{T}^{2}\right)$ using recent $\mathrm{LHCb}$ data.

\section{TMD factorisation and quarkonium production}

As clear from its name, TMD factorisation extends collinear factorisation by accounting for the parton transverse momenta, generally denoted $\boldsymbol{k}_{T}$. In the case of quarkonium-production processes, it is applicable either when a single quarkonium $\mathcal{Q}$ is produced with a transverse momentum, $P_{f T}$, typically smaller than half of its mass or when a quarkonium is produced in a set of two particles whose transverse momentum, $P_{f T}$, is also smaller than half of its invariant mass, $M_{f}$. In both cases, the observed final state should be colourless (see e.g. [8]). This imposes the quarkonia to be produced by Colour Singlet (CS) transitions (or equally speaking according to the CS Model (CSM)) and excludes the production in association with light hadrons, charm or beauty hadrons.

Under the TMD factorisation, the cross section for any gluon-fusion process can be expressed -up to corrections suppressed by powers of the observed-system transverse momentum- as a contraction and a convolution of a partonic short-distance contribution, $\mathcal{M}_{\mu \rho}$, with two gluon TMD correlators $\Phi_{g}$ evaluated at $\left(x_{1}, \boldsymbol{k}_{1 T}\right)$ and $\left(x_{2}, \boldsymbol{k}_{2 T}\right) . \mathcal{M}^{\mu \rho}$ is simply calculated in perturbative QCD through a series expansion in $\alpha_{s}$ [9] using Feynman graphs. Overall, we have

$$
\begin{aligned}
\mathrm{d} \sigma=\frac{(2 \pi)^{4}}{8 s^{2}} \int \mathrm{d}^{2} \boldsymbol{k}_{1 T} \mathrm{~d}^{2} \boldsymbol{k}_{2 T} \boldsymbol{\delta}^{2}\left(\boldsymbol{k}_{1 T}+\boldsymbol{k}_{2 T}-\boldsymbol{P}_{\boldsymbol{f} T}\right) \mathcal{M}_{\mu \rho}\left(\mathcal{M}_{v \sigma}\right)^{*} & \\
& \times \Phi_{g}^{\mu v}\left(x_{1}, \boldsymbol{k}_{1 T}\right) \Phi_{g}^{\rho \sigma}\left(x_{2}, \boldsymbol{k}_{2 T}\right) \mathrm{d} \mathcal{R},
\end{aligned}
$$

where $s=\left(P_{1}+P_{2}\right)^{2}$ is the hadronic centre-of-mass system (c.m.s.) energy squared, $P_{f}$ is the momentum of the observed final state, and where the phase-space element of the outgoing particles is denoted by $\mathrm{d} \mathcal{R}$. In addition, the gluon four-momenta $k_{i}$ are decomposed according to $k=x P+$ $k_{T}+k^{-} n$ [ $n$ refers to a light-like vector $\left(n^{2}=0\right)$ satisfying $\left.n \cdot P \neq 0\right], k_{T}^{2}=-k_{T}^{2}$ and $g_{T}^{\mu v}=g^{\mu v}-$ $\left(P^{\mu} n^{v}+P^{v} n^{\mu}\right) / P \cdot n$. 
In the case of unpolarised protons, the correlator can be parametrised $[5,10,11]$ in terms of the two aforementionned and independent TMDs, the unpolarised distribution $f_{1}^{g}\left(x, \boldsymbol{k}_{T}^{2}\right)$ and the distribution of linearly polarised gluons $h_{1}^{\perp g}\left(x, \boldsymbol{k}_{T}^{2}\right),{ }^{1}$

$$
\Phi_{g}^{\mu v}\left(x, \boldsymbol{k}_{T}\right)=-\frac{1}{2 x}\left\{g_{T}^{\mu v} f_{1}^{g}-\left(\frac{k_{T}^{\mu} k_{T}^{v}}{M_{p}^{2}}+g_{T}^{\mu \nu} \frac{\boldsymbol{k}_{T}^{2}}{2 M_{p}^{2}}\right) h_{1}^{\perp g}\right\},
$$

Following [14], the structure of the TMD differential cross section for an observed system of 2 colourless particles reads

$$
\begin{aligned}
& \frac{\mathrm{d} \sigma}{\mathrm{d} M_{f} \mathrm{~d} Y_{f} \mathrm{~d}^{2} \boldsymbol{P}_{\boldsymbol{f} T} \mathrm{~d} \Omega}=\mathcal{J} \times\left\{F_{1} \mathcal{C}\left[f_{1}^{g} f_{1}^{g}\right]+F_{2} \mathcal{C}\left[w_{2} h_{1}^{\perp g} h_{1}^{\perp g}\right]+\right. \\
& \left.\cos 2 \phi_{\mathrm{CS}}\left(F_{3} \mathcal{C}\left[w_{3} f_{1}^{g} h_{1}^{\perp g}\right]+F_{3}^{\prime} \mathcal{C}\left[w_{3}^{\prime} h_{1}^{\perp g} f_{1}^{g}\right]\right)+\cos 4 \phi_{\mathrm{CS}} F_{4} \mathcal{C}\left[w_{4} h_{1}^{\perp g} h_{1}^{\perp g}\right]\right\}
\end{aligned}
$$

where $\mathrm{d} \Omega=\mathrm{d} \cos \theta_{\mathrm{CS}} \mathrm{d} \phi_{\mathrm{CS}}, \theta_{\mathrm{CS}}$ and $\phi_{\mathrm{CS}}$ are the Collins-Soper (CS) angles [15] and $Y_{f}$ is the pair rapidity $-\boldsymbol{P}_{\boldsymbol{f} T}$ and $Y_{f}$ are defined in the hadron c.m.s. In the CS frame, the $\mathcal{Q}$ direction is along $\vec{e}=$ ( $\sin \theta_{\mathrm{CS}} \cos \phi_{\mathrm{CS}}, \sin \theta_{\mathrm{CS}} \sin \phi_{\mathrm{CS}}, \cos \theta_{\mathrm{CS}}$ ). The overall factor, $\mathcal{J}$, is specific to the mass of the finalstate particles and the analysed differential cross sections, and the hard factors $F_{i}$ do not depend on the rapidity of the pair nor on its transverse momentum.

For a single-particle (here quarkonium) production,

$$
\frac{\mathrm{d} \sigma}{\mathrm{d} Y \mathrm{~d}^{2} \boldsymbol{P}_{\boldsymbol{f} T}}=\mathcal{J}^{\prime} \times\left\{F_{1} \mathcal{C}\left[f_{1}^{g} f_{1}^{g}\right]+F_{2} \mathcal{C}\left[w_{2} h_{1}^{\perp g} h_{1}^{\perp g}\right]\right\}
$$

It is interesting to note that the TMDs appear in both above equations Eq. (2.3) and Eq. (2.4) in a factorised way from the hard-scattering coefficients through universal convolutions which read

$$
\mathcal{C}[w f g] \equiv \int \mathrm{d}^{2} \boldsymbol{k}_{1 T} \int \mathrm{d}^{2} \boldsymbol{k}_{2 T} \boldsymbol{\delta}^{2}\left(\boldsymbol{k}_{1 T}+\boldsymbol{k}_{2 T}-\boldsymbol{P}_{\boldsymbol{f} T}\right) w\left(\boldsymbol{k}_{1 T}, \boldsymbol{k}_{2 T}\right) f\left(x_{1}, \boldsymbol{k}_{1 T}^{2}\right) g\left(x_{2}, \boldsymbol{k}_{2 T}^{2}\right),
$$

where $w\left(\boldsymbol{k}_{1 T}, \boldsymbol{k}_{2 T}\right)$ are generic transverse weights and $x_{1,2}=\exp \left[ \pm Y_{f}\right] M_{f} / \sqrt{s}$. The $w\left(\boldsymbol{k}_{1 T}, \boldsymbol{k}_{2 T}\right)$ are identical for all the gluon-induced processes with unpolarised protons and can be found in [14]. For any process, one can show that $F_{2,3,4}^{\left({ }^{\prime}\right)} \leq F_{1}$.

The azimuthal modulations can be studied by evaluating [for $n=2,4$ ] weighted differential cross sections normalised to the azimuthally independent term like

$$
\frac{\int d \phi_{\mathrm{CS}} \cos n \phi_{\mathrm{CS}} \frac{d \sigma}{d M_{f} d Y_{f} d^{2} \boldsymbol{P}_{\boldsymbol{f} T} d \Omega}}{\int d \phi_{\mathrm{CS}} \frac{d \sigma}{d M_{f} d Y_{f} d^{2} \boldsymbol{P}_{\boldsymbol{f} T} d \Omega}} .
$$

One then gets that, in a single phase-space point, such $\cos 2 \phi_{\mathrm{CS}}$ modulations are proportional to $F_{3} \mathcal{C}\left[w_{3} f_{1}^{g} h_{1}^{\perp g}\right]+F_{3}^{\prime} \mathcal{C}\left[w_{3}^{\prime} h_{1}^{\perp g} f_{1}^{g}\right]$ and the $\cos 4 \phi_{\mathrm{CS}}$ modulations to $F_{4} \mathcal{C}\left[w_{4} h_{1}^{\perp g} h_{1}^{\perp g}\right]$.

\footnotetext{
${ }^{1}$ For gluon TMDs free from rapidity divergences from gauge links with paths (partly) along the light front $\xi \cdot n=0$, a soft factor $[1,12,13]$ is required. It does not play a role here and we therefore consider it as implicit.
} 


\section{Pseudoscalar-quarkonium production}

The case of the pseudoscalar-quarkonium production was first studied under the TMD factorisation by Boer and Pisano in Ref. [16]. It is very similar to that of $H^{0}$ boson and is particularly interesting since $F_{2}=-F_{1}$. From this, it follows that the $\boldsymbol{P}_{\boldsymbol{f} T}$ modulations generated by $h_{1}^{\perp g}\left(x, \boldsymbol{k}_{T}^{2}\right)$ are maximum. There are however some caveats. First, the domain where the TMD factorisation can be applied for $\eta_{c}$ production is admittedly very small since $P_{\eta_{c} T}$ should remain below $1 \sim 1.5 \mathrm{GeV}$. As for now, $\eta_{c}$ production was only studied by LHCb [17] for $P_{\eta_{c} T}$ larger than $6 \mathrm{GeV}$. Studies at lower $P_{\eta_{c} T}$ would require a specific effort on the triggers and on the background subtraction with however few hopes to reach $1 \sim 1.5 \mathrm{GeV}$. Studies in the fixed-target mode at $\sqrt{s_{N N}}=115 \mathrm{GeV}[18,19,20]$ with a smaller combinatorial background may be more promising. $\eta_{c}(2 S)$ production may be another option [21]. Slightly higher $P_{\eta_{c} T}$ may be dealt with by matching the TMD-factorised cross section with the collinearly factorised one with PDFs. However, this may preclude the extraction of experimental constraints on the TMDs [22]. Some of these caveats could be avoided by focusing one's effort on the $\eta_{b}$ case. However, it remains experimentally inaccessible at the LHC owing to its very small branchings to usable decay channels.

\section{4. $J / \psi+\gamma$ and $\Upsilon+\gamma$}

As we have just seen, the restriction $\boldsymbol{P}_{\boldsymbol{f} T} \lesssim M_{f} / 2$ is extremely detrimental for the $2 \rightarrow 1$ case. To bypass this constraint, it is thus expedient to consider 2-particle final states where $M_{f}$ can be tuned to reach the optimal kinematical range for $\boldsymbol{P}_{\boldsymbol{f} T}$ given the expected yields. Along these lines, we proposed [23] to study the associated production of $J / \psi+\gamma$ and $\Upsilon+\gamma$ at the LHC whose QCD corrections have been studied in the collinear factorisation in $[24,25,26]$.

If one is mainly interested in the extraction of $f_{1}^{g}$ via the $\boldsymbol{P}_{\boldsymbol{f} T}$ dependence of the cross section, this process is interesting since $F_{2}$ vanishes. As such, it is the complete opposite case compared to $\eta_{c}$ where the $\boldsymbol{P}_{\boldsymbol{f} T}$ modulation from $h_{1}^{\perp g}$ are maximum. As what concerns the azimuthal modulations, $F_{3}$ is power suppressed in $M_{f}$ while $F_{4}$ scales like $F_{1}$ offering interesting opportunities to extract $h_{1}^{\perp g}\left(x, \boldsymbol{k}_{T}^{2}\right)$ with existing LHC data by looking at $\cos 4 \phi_{\mathrm{CS}}$ modulations. The ratio $F_{4} / F_{1}$ is however significantly smaller than unity and, in practice, the $\cos 2 \phi_{\mathrm{CS}}$ modulations are not necessarily much smaller. Studies in the fixed-target mode with a LHCb-like detector are feasible and may bring about constraints at $x$ as high as $0.5[18,27]$.

Similarly, $J / \psi$ and $\Upsilon$ can be gluo-produced in association with an off-shell photon or a $Z$ boson. We studied this case in Ref. [14]. The rates are however probably too small [28], even at the LHC, for azimuthal-modulation studies. In addition, there may be a significant contamination by Double Parton Scatterings (DPSs) [29, 30] in the currently accessible region at the LHC [31].

\section{Vector-quarkonium-pair production}

The third case we discuss here is that of the production of a pair of $J / \psi$ or $\Upsilon$. As compared to the former processes, these reactions are extremely appealing for the following reasons:

- the hard-scattering coefficients $F_{i}$ are extremely favourable to the observation of azimuthal modulations [32];

- significant data sample have already been collected at the LHC and the Tevatron at different invariant masses [33, 34, 35, 36, 37]; 
- the DPS contamination is probably on the order of $10 \%[38,36]$, where the azimuthal modulations are expected to be the largest;

- the contamination from the non-TMD-factorising colour-octet transitions is also negligible -as expected from its $v^{8}$ suppression- in this region [39, 40, 38].

As what concerns the hard-scattering coefficients, it is interesting to look at them both in the large and small $M_{f}=M_{\mathcal{Q}}$ limits. Indeed, when $M_{\mathcal{Q} Q}$ becomes much larger than the quarkonium mass, $M_{\mathcal{Q}}$, one finds [32] that, for $\cos \theta_{\mathrm{CS}} \rightarrow 0$ (i.e. small $\Delta y$ ),

$$
F_{4} \rightarrow F_{1}, \quad F_{2} \rightarrow \frac{81 M_{\mathcal{Q}}^{4} \cos \theta_{\mathrm{CS}}^{2}}{2 M_{\mathcal{Q} \mathcal{Q}}^{4}} \times F_{1}, \quad F_{3} \rightarrow \frac{-24 M_{\mathcal{Q}}^{2} \cos \theta_{\mathrm{CS}}^{2}}{M_{\mathcal{Q} \mathcal{Q}}^{2}} \times F_{1}
$$

The result $F_{4} \rightarrow F_{1}$ in this limit is thus far unique among all the gluon-TMD-sensitive processes, making di-quarkonium production the most sensitive probe of $h_{1}^{\perp g}$.
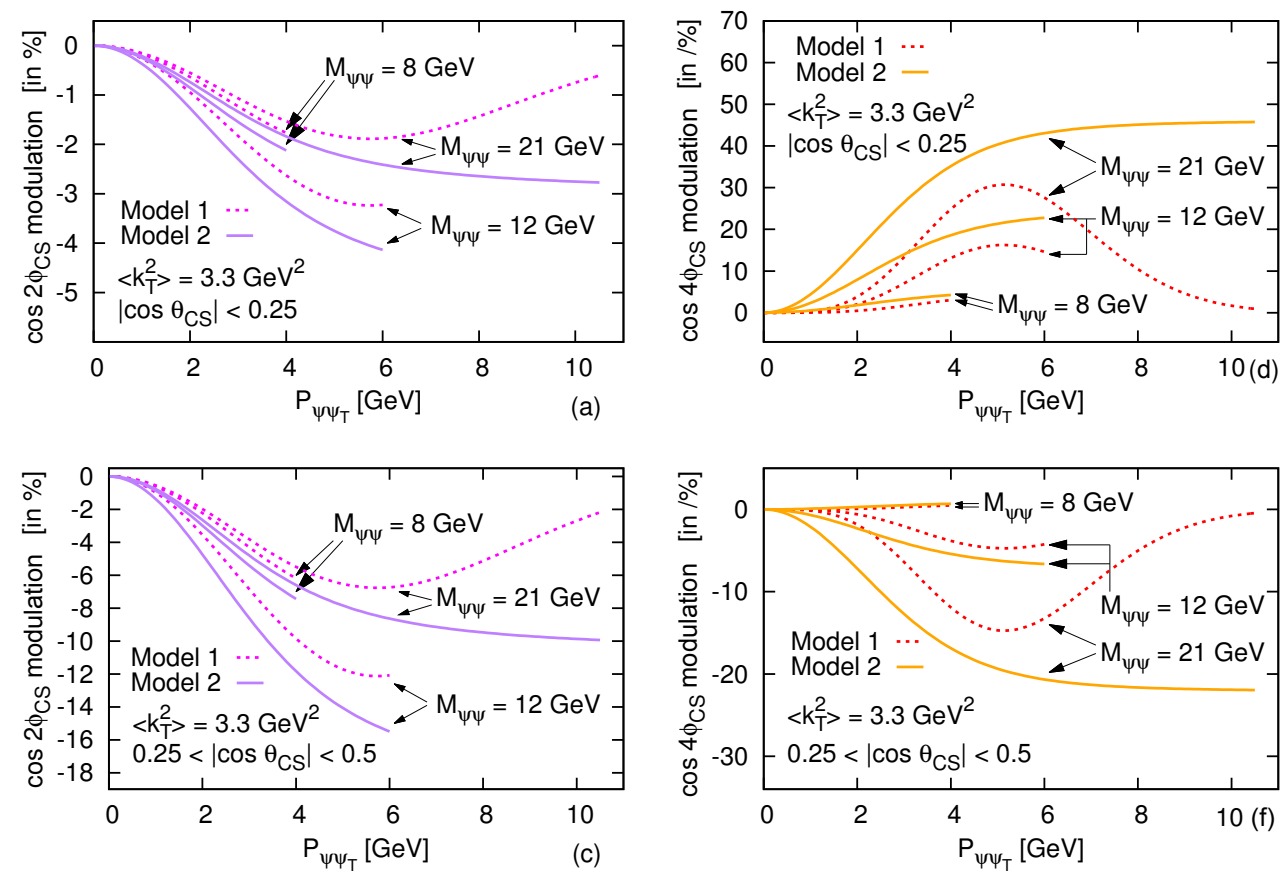

Figure 1: $\cos n \phi_{\mathrm{CS}}$ modulations for $n=2,4$ computed for $\left|\cos \theta_{\mathrm{CS}}\right|<0.25$ and for $0.25<\cos \theta_{\mathrm{CS}}<0.5$ for both our models of $h_{1}^{\perp g}$, for 3 values of $M_{\mathcal{Q Q}}(8,12$ and $21 \mathrm{GeV})$ relevant respectively for the LHCb [37], CMS [35] and ATLAS [36] kinematics. The spectra are plotted up to $M_{\mathcal{Q Q}} / 2$. Our results do not depend on $Y_{\mathcal{Q Q}}$.

For $M_{f}$ close to $2 M_{\mathcal{Q}}$-its minimum value, $F_{2} \rightarrow 3 / 787 \times F_{1}$ and $F_{3,4} \rightarrow 0$. Even though $F_{2}$ is not strictly zero, like in the $\mathcal{Q}+\gamma$ case, it is always very small and generates negligible modulations to the $\boldsymbol{P}_{\boldsymbol{f} T}$-differential cross section. Based on this, we performed in Ref. [32] the first extraction of $f_{1}^{g}$ using the latest LHCb data [37]. Assuming a Gaussian $\boldsymbol{k}_{T}$ dependence we obtained $\left\langle\boldsymbol{k}_{T}^{2}\right\rangle=3.3 \pm 0.8 \mathrm{GeV}^{2}$ which encapsulates both non-perturbative and perturbative effects since the scale relevant for such data sample is on the order of $8 \mathrm{GeV}$. Using this $\left\langle\boldsymbol{k}_{T}^{2}\right\rangle$ value and modellings of $h_{1}^{\perp g}$ such as the Gaussian form of Ref. [6] (Model 1) or saturating the positivity bound [5, 41] 
(Model 2), we obtained the $\cos n \phi_{\mathrm{CS}}$ modulations shown in Fig. 1 where we note that the $\cos 2 \phi_{\mathrm{CS}}$ modulations are becoming larger for increasing $\cos \theta_{\mathrm{CS}}$.

\section{Conclusions}

Quarkonium hadroproduction offers interesting possibilities to study gluon TMDs which only start to be investigated. We briefly reviewed here the cases of single-pseudoscalar-quarkonium production, vector-quarkonium pair production and associated production of a vector quarkonium with a photon or a $Z^{0}$ boson. The most promising case is that of $J / \psi+J / \psi$ which we expect to be studied soon at the LHC along the lines presented here, i.e. with a dedicated measurement of the $\cos 2 \phi_{\mathrm{CS}}$ and $\cos 4 \phi_{\mathrm{CS}}$ modulations.

These would complement -with a clean access to $f_{1}^{g}$ and $h_{1}^{\perp g}$ - future target-spin asymmetry studies (see e.g. $[42,18]$ ) to measure the gluon Sivers function $f_{1 T}^{\perp g}$ as well as distributions of linearly-polarised gluons in a transversely polarised proton, $h_{1 T}^{g}$ and $h_{1 T}^{\perp g}$, allowing for a complete gluon tomography of the proton.

\section{References}

[1] J. Collins, Camb. Monogr. Part. Phys. Nucl. Phys. Cosmol. 32, 1 (2011).

[2] S. M. Aybat, T. C. Rogers, Phys. Rev. D83, 114042 (2011).

[3] M. G. Echevarria, A. Idilbi, I. Scimemi, JHEP 07, 002 (2012).

[4] R. Angeles-Martinez, et al., Acta Phys. Polon. B46, 2501 (2015).

[5] P. J. Mulders, J. Rodrigues, Phys. Rev. D63, 094021 (2001).

[6] D. Boer, W. J. den Dunnen, C. Pisano, M. Schlegel, W. Vogelsang, Phys. Rev. Lett. 108, 032002 (2012).

[7] D. Boer, W. J. den Dunnen, C. Pisano, M. Schlegel, Phys. Rev. Lett. 111, 032002 (2013).

[8] D. Boer, Few Body Syst. 58, 32 (2017).

[9] J. P. Ma, J. X. Wang, S. Zhao, Phys. Rev. D88, 014027 (2013).

[10] S. Meissner, A. Metz, K. Goeke, Phys. Rev. D76, 034002 (2007).

[11] D. Boer, et al., JHEP 10, 013 (2016).

[12] M. G. Echevarria, A. Idilbi, I. Scimemi, Phys. Lett. B726, 795 (2013).

[13] M. G. Echevarria, T. Kasemets, P. J. Mulders, C. Pisano, JHEP 07, 158 (2015). [Erratum: JHEP05,073(2017)].

[14] J.-P. Lansberg, C. Pisano, M. Schlegel, Nucl. Phys. B920, 192 (2017).

[15] J. C. Collins, D. E. Soper, Phys. Rev. D16, 2219 (1977).

[16] D. Boer, C. Pisano, Phys. Rev. D86, 094007 (2012).

[17] R. Aaij, et al., Eur. Phys. J. C75, 311 (2015).

[18] C. Hadjidakis, et al., arXiv:1807.00603 (2018).

[19] S. J. Brodsky, F. Fleuret, C. Hadjidakis, J. P. Lansberg, Phys. Rept. 522, 239 (2013).

[20] J. P. Lansberg, S. J. Brodsky, F. Fleuret, C. Hadjidakis, Few Body Syst. 53, 11 (2012).

[21] J.-P. Lansberg, H.-S. Shao, H.-F. Zhang, arXiv:1711.00265 .

[22] M. G. Echevarria, T. Kasemets, J.-P. Lansberg, C. Pisano, A. Signori, Phys. Lett. B781, 161 (2018).

[23] W. J. den Dunnen, J. P. Lansberg, C. Pisano, M. Schlegel, Phys. Rev. Lett. 112, 212001 (2014).

[24] R. Li, J.-X. Wang, Phys. Lett. B672, 51 (2009).

[25] J. P. Lansberg, Phys. Lett. B679, 340 (2009).

[26] R. Li, J.-X. Wang, Phys. Rev. D89, 114018 (2014).

[27] J. P. Lansberg, et al., EPJ Web Conf. 85, 02038 (2015).

[28] B. Gong, J.-P. Lansberg, C. Lorce, J. Wang, JHEP 03, 115 (2013).

[29] J.-P. Lansberg, H.-S. Shao, JHEP 10, 153 (2016).

[30] J.-P. Lansberg, H.-S. Shao, N. Yamanaka, Phys. Lett. B781, 485 (2018).

[31] G. Aad, et al., Eur. Phys. J. C75, 229 (2015).

[32] J.-P. Lansberg, C. Pisano, F. Scarpa, M. Schlegel, Phys. Lett. B784, 217 (2018)

[33] R. Aaij, et al., Phys. Lett. B707, 52 (2012).

[34] V. M. Abazov, et al., Phys. Rev. D90, 111101 (2014).

[35] V. Khachatryan, et al., JHEP 09, 094 (2014).

[36] M. Aaboud, et al., Eur. Phys. J. C77, 76 (2017).

[37] R. Aaij, et al., JHEP 06, 047 (2017).

[38] J.-P. Lansberg, H.-S. Shao, Phys. Lett. B751, 479 (2015).

[39] P. Ko, C. Yu, J. Lee, JHEP 01, 070 (2011).

[40] Y.-J. Li, G.-Z. Xu, K.-Y. Liu, Y.-J. Zhang, JHEP 07, 051 (2013).

[41] S. Cotogno, T. van Daal, P. J. Mulders, JHEP 11, 185 (2017).

[42] D. Kikola, et al., Few Body Syst. 58, 139 (2017). 